\title{
Numerical Prediction of Smoke Detector Activation Accounting for Aerosol Characteristics
}

\author{
WEI ZHANG, MICHAEL KLASSEN, and RICHARD ROBY \\ Combustion Science \& Engineering, Inc. \\ 8940 Old Annapolis Road, Suite L \\ Columbia, MD, 21045 USA
}

\begin{abstract}
This study integrates a time lag dynamic response algorithm that describes the response time of a smoke detector and a smoke aerosol tracking procedure into a Large Eddy Simulation (LES) fire model. The LES fire model predicts the smoke concentration adjacent to the detector while a time lag dynamic response algorithm and describes the transport of smoke into the sensing chamber. The smoke particle number concentrations in the sensing chamber are calculated based on the initial particle size and distribution. Experimental data from a standard fire test was used for validation. Overall, reasonable results were obtained when comparing the predictions to the experimental results.
\end{abstract}

KEYWORDS: aerosol behavior, detection, modeling, CFD

\section{NOMENCLATURE LISTING}

\begin{tabular}{|c|c|c|c|}
\hline$N_{0}$ & Initial particle number concentration & $T_{o}$ & Ambient temperature (K) \\
\hline$Y$ & Mass fraction of smoke $\left(\mathrm{mg} / \mathrm{m}^{3}\right)$ & $t$ & Time (s) \\
\hline$d g$ & Geometric mean particle diameter(m) & $L p$ & Path length (m) \\
\hline$\sigma_{g}$ & Geometric standard deviation & $K_{n 0}$ & $\begin{array}{l}\text { Knudsen number } \\
\text { Constant of coagulation }\end{array}$ \\
\hline$\rho_{p}$ & Particle density $\left(\mathrm{kg} / \mathrm{m}^{3}\right)$ & $A_{\text {con }}$ & factor \\
\hline$V p$ & Total volume of the particle & & \\
\hline$N(v)$ & Particle size distribution & $\lambda$ & Mean free path (m) \\
\hline$V g$ & Geometric mean particle volume & $\kappa_{b}$ & Boltzman constant \\
\hline$V$ & Total volume & $v_{\text {sgs }}$ & Turbulent viscosity \\
\hline$N$ & Particle number concentration $\left(\mathrm{N} / \mathrm{cm}^{3}\right)$ & $\kappa$ & Constant of proportionality \\
\hline & Temperature (K) & & particles \\
\hline
\end{tabular}

\section{INTRODUCTION}

Early detection of fire plays an important role in the life safety of building occupants. Smoke detection is a common method for early notification of a fire. Basically, there are two types of smoke detectors that are commonly used in residential and industrial situations: ionization and photoelectric. The operation of these detectors depends on the physical properties of smoke aerosol particles in the sensing chamber, which include the particle numbers and size distribution. It has been shown that the ionization-type detector is more sensitive to small smoke particles (smaller than $0.3 \mu \mathrm{m}$ ), and a photoelectric-type detector is more sensitive to larger smoke particles (larger than $0.3 \mu \mathrm{m}$ ) [1]. Therefore, the methods to properly model the time lag dynamic response in the smoke detector and the aerosol behavior in the sensing chamber has become a challenge in order to better 
understand and evaluate smoke detector performance, the design of alarm systems and for overall fire safety strategies.

It is well known that CFD (Computational Fluid Dynamics) fire modeling can predict detailed velocity and smoke concentration in compartments. In particular, LES can properly predict velocity fields close to the walls when an adequate wall function is used, and the resolution of the grid can reasonably resolve dimensions comparable to the size of the smoke detector. On the other hand, since the detector activation depends on the number of the smoke particles and the particle size distribution in the sensing chamber, the smoke particle transport within the sensing chamber must be resolved. However, the relative dimensions of the sensing chambers are too small in comparison with those of the fire room size; it is computationally too costly to sufficiently resolve the grid as fine as necessary to establish the transport of smoke particles within the sensing chamber. Therefore, implementation of an analytic model with reasonable assumptions will be a better choice for modeling of the transport of smoke particles in the sensing chamber. This model will consist of the two major parts: (1) the modeling of the entry time lag as a detector geometry effect and (2) the modeling of the aerosol particle behavior in the sensing chamber.

In order to model the smoke entry time lag of the detector as a detector geometry effect, Heskestad [2] proposed a one characteristic time detector model. This approach is adequate at sufficiently high velocities (Newman [3]), but it is lacking when the velocity is low (Cleary et al. [4]). In order to deal with the full range of velocity conditions, Newman [5] and Cleary et al. [4] suggested a two characteristic times detector model. Zhang et al. [6] implemented the two characteristic times model into a Large Eddy Simulation (LES) fire modeling, and reasonable results were obtained when comparing the predictions to the experimental results. However, a model that considers time lag only cannot represent the effect of the particle number and size distribution in the sensing chamber, which is in general, associated with different fuels or fire types (such as a flaming or smoldering fire). On the other hand, Mirme et al. [7] reported the measurements of the aerosol particle number concentration and the size distribution at the detector location of the standard test-fires (EN54/9,TF1 5). Farouk et al. [8] implemented and predicted the particle number concentration of smoke aerosol of an open plastic fire (EN54/9,TF4) at the detector location by using an early version of FDS and a simplified aerosol coagulation process. The comparison of the predicted results and experimental data show that the aerosol number concentration of smoke was over predicted at the detector location. Recently, Rexfort [9] predicted the particle number concentration and the size distributions of a cotton wick fire (EN54/9,TF3) as well as an n-heptane fire (EN54/9, TF5) at the detector location by using an improved coagulation process model. The predicted results give reasonable agreement with experimental data. However, since the smoke entry time lag model had not yet been correctly included in these works, it is still lacking on the particle number and size distribution in sensing chamber and the application of the modeling for the residential and industrial smoke detector in realistic situations.

The studies described above indicate that it is important not only to establish the transport time lag between the fire and the smoke detector but also to properly establish the transfer of smoke particles from the outside of the detector into the sensing chamber. None of the above studies have attempted to combine both processes in order to predict detector activation. This can be done using a CFD approach because it provides detailed local and temporal resolutions of the velocity fields close to the detector as well as a 
precise estimation of the local concentration of smoke and particle behavior. In this study, a dynamic response of the smoke entry time lag model combined with an aerosol behavior tacking procedure was integrated into a LES fire modeling. The purpose of this study is not only to predict the smoke transport time lag between the fire smoke and the detector sensing chamber, but also establish the aerosol behavior in the detector sensing chamber. Two standard fire testing cases have been used for validation: (1) an Open Plastic Fire (EN54/9, TF4 [10]) used for standard testing of the smoke detector activation, and (2) a standard test for a single and multiple station smoke detectors with a polystyrene fire source (UL217/test D [11]).

\section{MODELING OF SMOKE DETECTOR}

\section{Smoke Entry Time Lag Model}

Ionization and photoelectric detectors use different principles of operation to sense the particles of the smoke. However for modeling of the smoke entry time lag purposes, both can be thought of as a sensing chamber with an entry passage that exhibits flow resistance. Therefore, given a smoke concentration outside the detector, the response of the detector can be established through a modeling procedure. The two characteristic times that describe smoke model detector activation are: (1) the dwell time $\delta \mathrm{t}$ and the mixing time $\tau$. According to Cleary et al. [4], the dwell time $\delta$ t and the mixing time $\tau$ both can be represented as a power law dependence on velocity as follows:

$$
\begin{aligned}
& \delta t=\alpha_{1} u^{-\beta_{1}} \\
& \tau=\alpha_{2} u^{-\beta_{2}}
\end{aligned}
$$

where $\alpha_{1}, \beta_{1}, \alpha_{2}, \beta_{2}$ are proportionality constants of the dwell time and mixing time that account for the detector geometry. The change of the mass of smoke in the sensing chamber $Y_{o}$ can be found by solving the following equation.

$$
\frac{\partial Y_{o}}{\partial t}=\frac{Y_{e}(t-\delta t)-Y_{o}(t)}{\tau}
$$

where $\mathbf{e}$ represents the entrance of the model detector and $\mathbf{o}$ represents the sensing chamber. $Y_{e}$ is the mass fraction of smoke outside of the model detector, and $Y_{o}$ is the mass fraction of smoke inside of the sensing chamber. If the initial mass fraction of smoke in the sensing chamber is zero, the mass fraction of smoke in the sensing chamber can be obtained by integrating equation (3) to obtain [6]:

$$
Y_{0}(t)=\exp \left(-\int_{0}^{t} \frac{1}{\tau} d t^{\prime}\right)\left\{\int_{0}^{t}\left(\frac{1}{\tau} \cdot \exp \left(\int_{0}^{t} \frac{1}{\tau} d t^{\prime}\right) \cdot Y_{e}\left(t^{\prime}-\delta t\right)\right) d t^{\prime}\right\}
$$

The predicted mass fraction of smoke in the sensing chamber $Y_{o}$ can be expressed as an obscuration per unit length as follows:

$O_{m}=\left(1-\frac{I}{I_{0}}\right) \times 100=\left(1-\exp \left(-\kappa Y_{o} L_{P}\right)\right) \times 100$ 
where the product $\kappa Y o$ is termed the extinction coefficient, $L_{P}$ is the path length in meters and $\kappa$ is a constant of proportionality termed the specific extinction $\left(\mathrm{m}^{2} / \mathrm{g}\right)$ [12]. For most flaming fuels, the value of $7.6 \mathrm{~m}^{2} / \mathrm{g}$ was used as a default of FDS4.0.

It is assumed that when the obscuration $(\% / \mathrm{m})$ in sensing chamber is larger than a threshold, the smoke detector will activate. The threshold can be set from $1.6 \% / \mathrm{m}$ to $14 \% / \mathrm{m}(0.5 \% / \mathrm{ft}$ to $4 \% / \mathrm{ft})$ range, according to the standard setting of the detector devices commonly provided by manufacturers. In this study, the value of $1.6 \% / \mathrm{m}$ was used as a threshold.

Note that the activation model summarized by Eq. 3, 4, and 5 is slightly more complicated than one suggested by Heskestad [2] and others [1]. The simpler model [2] considers only one characteristic time that is inversely proportional to the free-stream velocity, which corresponds to one characteristic length $\mathrm{L}$. Therefore, when the four empirical parameters are set as $\alpha_{1}=0, \alpha_{2}=L, \beta_{1}=\beta_{2}=-1$, the model reverts to the same form as the simpler one.

\section{Aerosol Behavior in and Around Detector Model}

Due to the different mechanisms of the physical phenomenon of the smoke particles (such as coagulation; condensation; evaporation and agglomeration etc.), detailed aerosol behavior can be obtained by implementing a general dynamic equation for the particle size distribution function into a CFD code, in which the size distribution of the particles changes dynamically. However, as mentioned previously, it is computationally too costly to sufficiently resolve the grid to establish the transport of smoke particles within the sensing chamber by CFD. Therefore, it is necessary to develop a simplified analytic solution including important mechanisms that most effect the smoke particle size change in the smoke detector activation modeling.

The starting point of the aerosol behavior model is based on the mass fraction of smoke and velocity in the detector location and sensing chamber. According to aerosol theory [24], the mass fractions of smoke are associated with the particle number concentration (PNC), the geometric mean particle diameter (GMD) and the geometric standard deviation (GSD). In order to convert the mass fraction of smoke into these three parameters (PNC, GMD, GSD), the particle size distribution should be given in advance. In general, the particle size distribution can be given as follows [13]:

$$
n(v)=\frac{N_{0}}{3 \sqrt{2 \pi} \cdot \ln \left(\sigma_{g}\right) \cdot V} \exp \left\{-\frac{\ln ^{2}\left(\frac{V}{V_{g}}\right)}{18 \ln ^{2} \sigma_{g}}\right\}
$$

Where, $\mathrm{Vg}$ is the geometric mean particle volume and $\sigma_{\mathrm{g}}$ is the geometric standard deviation of the particle size distribution. And the initial particle number concentration can be obtained as follows:

$$
N_{o}=\frac{Y}{\rho_{P} \cdot V_{g}} \exp \left(-\frac{9}{2} \ln ^{2} \sigma_{g}\right)
$$


Where, the geometric mean volume: $V_{g}=\frac{1}{6} \pi d_{g}{ }^{3}$, and $\mathrm{d}_{\mathrm{g}}$ is the GMD. As can be noted, if the geometric mean particle diameter (GMD) and the geometric standard deviation (GSD) are known, the initial particle number concentration can be determined by Eq. 7 .

\section{Smoke Aerosols with Brownian Coagulation Process}

The particle size distribution of smoke aerosols is dynamic. This characteristic of smoke aerosols is due to Brownian motion of the particles that causes them to collide and stick together, known as a coagulation process. Coagulation has the effect of decreasing the number concentration. Other physical processes that affect the particles include condensation of vapor onto existing particles, evaporation of the volatile component of the smoke, and agglomeration and deposition onto the walls. However, it is believed that the most important physical process to influence the particle size distribution of the smoke is the coagulation process [14]. In this study, the coagulation process was focused on.

The integrating-differential equation that governs the continuous size distribution of coagulating aerosol can be given as follows [14]:

$\frac{\partial n(v, t)}{\partial t}=\frac{1}{2} \int_{0}^{v} \beta(v-\bar{v}, \bar{v}) n(v-\bar{v}, t) n(\bar{v}, t) d \bar{v}-n(v, t) \int_{0}^{\infty} \beta(v, \bar{v}) n(\bar{v}, t) d \bar{v}$

where $n(v, t)$ is the particle size distribution function, $\mathrm{v}$ and $\bar{v}$ are two different particle volumes, $\beta(v, \bar{v})$ is the collision kernel for these two particle volumes. The first term on the right side of Eq. 8 represents the production rate of particle size $\mathrm{v}$ by collision of particles of size $v-\bar{v}$ and $\bar{v}$ and the second term on the right size represents the disappearance rate of particles with volume v. The particle size covered by Eq. 8 can be divided into three regimes by using the Knudsen number, which is the ratio of the mean free path of an atom in the gas and the radius of the particles. If the Knudsen number is less than 0.10 , this is known as the continuum regime. If the Knudsen number is between 0.1 and 10, this is known as the slip regime and if the Knudsen number is large than 10, this is known as the free molecule regime. Experiments have shown that the smoke aerosols lie in the slip regime and close to the continuum regime (Knudsen number less than 5.0) [14]. Therefore, only the continuum regime and the slip regime were considered in this study.

Considering Brownian motion within continuum and slip regime, the collision $\operatorname{kernel} \beta(\mathrm{v}, \overline{\mathrm{v}})$ can be written as follows [13]:

$\beta(v, \bar{v})=K_{C} \cdot\left(v^{\frac{1}{3}}+\bar{v}^{\frac{1}{3}}\right)\left(\frac{C(v)}{v^{1 / 3}}+\frac{C(\bar{v})}{\bar{v}^{1 / 3}}\right)$

where, $\mathrm{C}(\mathrm{v})$ is the slip correction factor that take into account the effects of the gas slip for small particles. When the Knudsen number is less than 5.0, the following form is used to represents these effects [15]

$C(v)=1.0+A_{\mathrm{CON}} \cdot K n_{0}$

Here, $A_{C O N}$ is constant, $\mathrm{Kn}_{0}$ is initial Knudsen number $\left(\mathrm{Kn}_{0}=2 \lambda / \mathrm{d}_{\mathrm{g}}\right)$, and $\lambda$ is the mean free path. When the particles sizes are within the continuum regime, $A_{\mathrm{CON}}$ is set to zero. 
For the slip regime, testing and analysis has found that $A_{C O N}=0.1591$ is a proper constant for smoke aerosol particle motions [9].

Using Eq. 10, an analytical solution for Eq. 8 was obtained by Lee et al. [13]. As the particle number concentration decreases $\left(\mathrm{N} / \mathrm{N}_{0}\right)$, Lee et al. found:

$$
\begin{aligned}
& K_{C} N_{0} t=\frac{3}{a}\left[\frac{1}{3}\left\{\left(N / N_{0}\right)^{-1}-1\right\}-\left(\frac{b}{2 a}\right)\left\{\left(N / N_{0}\right)^{-\frac{2}{3}}-1\right\}+\left(\frac{b}{a}\right)^{2}\left\{\left(N / N_{0}\right)^{-\frac{1}{3}}-1\right\}-F_{S}\right] \\
& F_{S}=\left(\frac{b}{a}\right)^{3} \cdot \operatorname{Ln}\left\{\frac{1+(b / a) \cdot\left(N / N_{0}\right)^{1 / 3}}{(1+(b / a)) \cdot\left(N / N_{0}\right)^{1 / 3}}\right\}, a=1+\exp \left(Z_{0}\right), Z_{0}=\ln ^{2} \sigma_{g_{0}} \\
& b=A_{C O N} \cdot K_{n 0}\left\{\exp \left(\frac{Z_{0}}{2}\right)+\exp \left(\frac{5 Z_{0}}{2}\right)\right\},
\end{aligned}
$$

Here, $K_{C}=2 \kappa_{b} T /\left(3 v_{\text {sgs }}\right)$ where $\kappa_{b}$ is Boltzmann constant, $T$ is the temperature and $v_{\mathrm{sgs}}$ is turbulent viscosity.

For the geometric mean particle volume, we have:

$\frac{v_{g}}{v_{g 0}}=\frac{\exp \left((9 / 2) Z_{0}\right)\left(N / N_{0}\right)^{-1}}{\left[2 c+\left(N / N_{0}\right)\left\{\exp \left(9 Z_{0}\right)-2 c\right\}\right]^{1 / 2}}$

$c=\frac{a+b \cdot \exp \left(-3 Z_{0}\right)\left(N / N_{0}\right)^{1 / 3}}{a+b \cdot\left(N / N_{0}\right)^{1 / 3}}$

For the geometric standard deviation, we have:

$Z=\ln ^{2} \sigma_{g}=\frac{1}{9} \ln \left[2 c+\left(N / N_{0}\right)\left\{\exp \left(9 Z_{0}\right)-2 c\right\}\right]$

It should be noted that Eq. 11 is an implicit equation as a function of the time that requires an iterative methodology to solve. However, once the particle number concentration decrease $\left(\mathrm{N} / \mathrm{N}_{\mathrm{O}}\right)$ is found at a give time, the geometric mean particle volume (Eq. 12) and the geometric standard deviation (Eq. 13) can be obtained directly.

\section{The Implementation of the Smoke Detector Model}

At the smoke detector location, it is assumed that LES grid resolution can reasonably approximate the size of the smoke detector. Thus the mass fraction of smoke and velocity at that location can be determined from the solutions produced by FDS [17,20,21]. The mass fraction inside of the sensing chamber is then determined by Eq. 4. In order to obtain a solution for Eq. 4, a numerical integration using an extended trapezoidal rule was applied. Two subroutines were developed, one for calculation of the time function $\left(\int_{0}^{t} \frac{1}{\tau} \mathrm{dt}^{\prime}\right)$

and another to calculate the expression presented in Eq. 4. When the transient time is smaller then dwell time $\delta \mathrm{t}$, the mass fraction of smoke has not entered the sensing chamber yet, and the $Y_{o}$ inside of sensing chamber is established at zero. The numerical integration of Eq. 4 will be initiated when the transient time is larger then dwell time. The calculated $\mathrm{Y}_{\mathrm{O}}$ will be converted to the obscuration $(\% / \mathrm{m})$ using Eq. 5. It is assumed that when the obscuration $(\% / \mathrm{m})$ in sensing chamber is larger than the alarm threshold, the smoke detector will activate. The alarm threshold can be set to any value, however this value is typically $1.6 \% / \mathrm{m}$ to $14 \% / \mathrm{m}(0.5 \% / \mathrm{ft}$ to $4 \% / \mathrm{ft})$, as provided by smoke detector manufacturers. This study used $1.6 \%$ as an alarm threshold. 
The initial particle number concentration will be calculated by Eq. 7 with the initial geometric mean particle diameter and the geometric standard deviation. Due to the smoke particle coagulation process, the particle number concentration is obtained by solving the Eq. 11 with the Newton-Raphson method. Finally, a new geometric mean particle diameter and the geometric standard deviation are obtained from Eqs. 12 and 13.

\section{THE APPLICATIONS OF THE SMOKE DETECTOR ACTIVATION MODEL}

\section{A Single/Multiple Station Smoke Detectors Test Case (UL 217 Test Case D)}

UL 217 [11] provides a rigid setup with multiple locations for photocell assemblies and smoke detectors. UL217 prescribes a test scenario in which the smoke must reach each of the sampling locations within the test room during a certain window of elapsed time. This test case is often used for smoke detector testing or modeling validation [20]. A UL217 test D (Polystyrene fire) was used for verification of the smoke built up in this study. To determine the heat release rate as an input data in fire modeling, oxygen consumption calorimetry was used to find the heat release profile of the polystyrene test sample used in UL217 test D. The profile of HRR was determined by burning a prescribed sample of foam polystyrene type packing material with density between $24 \sim 32 \mathrm{~kg} / \mathrm{m} 3$, and no flame inhibitor, under a collection hood with oxygen sampling [20].

The geometry of the fire test room can be found from [11]. The room is $10.9 \mathrm{~m}$ (long) by $6.7 \mathrm{~m}$ (wide) by $3.1 \mathrm{~m}$ (height), with a smooth ceiling with no physical obstructions. The test is to be conducted in an ambient temperature between $20-25^{\circ} \mathrm{C}$. Two detectors are to be tested on a ceiling panel, and another two detectors are to be tested on each sidewall panel. The calculation grid was $80(x) \times 40(y) \times 30(z)$ for the model and the runtime was 120 seconds. A value of 0.164 was selected for soot yield of the Polystyrene [20].

\section{Results and Discussion}

Since the smoke properties of primary interest to the fire community are obscuration $(\% / \mathrm{m})$, the mass concentration of smoke in and around detector was calculated by FDS combined with the time lag detector model, which was converted into obscuration $(\% / \mathrm{m})$ by using equation (5).

Table 1 shows the comparison of the modeling results and the UL 217 requirement. Table 1a shows the smoke arrival time and the detector activation time modeled by FDS. It can be noted that all of the predicted times for smoke arrival are around 42 to 44 seconds, which satisfied the UL217 requirement for ceiling detectors but is slightly higher than the requirement for sidewall detectors. This over prediction also can be found from the modeling of the detector activation time, in which the ceiling location detectors activation were predicted at 65 seconds, and the sidewall detectors activation was predicted at 56 58 seconds, which indicted that the smoke concentration was too high at the wall. Table $1 \mathrm{~b}$ shows the predictions of time for $33 \%$ obscuration (per meter) to occur. The model predicted this time to be within 70(s) 76(s) at all detector locations, which satisfied the UL217 requirement for both ceiling and wall detectors. This finding provides confidence in the smoke properties chosen to represent polystyrene in the model.

Tables 1c and 1d demonstrate the UL 217 requirement that the obscuration level be maintained at $33 \% / \mathrm{m} \sim 43 \% / \mathrm{m}$ at the ceiling locations of the detectors from 90 seconds to the end. Similarly, the obscuration level at the location of the sidewall detectors should remain between $33 \% / \mathrm{m} \sim 56 \% / \mathrm{m}$ obscuration from 80 seconds to the end. Obscuration 
predictions at both locations indicate reaching the $33 \% / \mathrm{m}$ obscuration level slightly early, with the ceiling detector location getting there at 75 seconds and sidewall detector location reaching that level at 70 seconds. Over all, although there was a slight over prediction of the smoke built up, FDS modeling obtained reasonable results in comparison with UL217 requirements at both ceiling and wall side detector locations.

Table 1. Results of the UL217 test and model.

(a) Smoke arrival shall occur.

\begin{tabular}{|l|l|l|l|}
\hline & UL217(s) & \multicolumn{1}{|c|}{ FDS4.0 } & Predicted activation time(s) \\
\hline Ceiling Detector A & $35 \sim 45$ & 44.0 & 65.6 \\
\hline Ceiling Detector B & $35 \sim 45$ & 43.8 & 65.2 \\
\hline Sidewall Detector A & $25 \sim 35$ & 42.0 & 56.5 \\
\hline Sidewall Detector B & $25 \sim 35$ & 58.1 & 42.5 \\
\hline
\end{tabular}

(b) $33 \% / m$ obscuration shall occur.

\begin{tabular}{|l|l|l|}
\hline & UL217 requires(s) & FDS4.0(s) \\
\hline Ceiling Detector A & $70 \sim 90$ & $75.7 \sim 76.0$ \\
\hline Ceiling Detector B & $70 \sim 90$ & $75.8 \sim 76.2$ \\
\hline Sidewall Detector A & $60 \sim 80$ & $70.5 \sim 73.0$ \\
\hline Sidewall Detector B & $60 \sim 80$ & $70.2 \sim 70.7$ \\
\hline
\end{tabular}

(c) $33 \% / \mathrm{m} \sim 43 \% / \mathrm{m}$ obscuration shall remain.

\begin{tabular}{|l|l|l|}
\hline & UL217 requires(s) & FDS4.0(s) \\
\hline Ceiling Detector A & $90 \sim 120$ (end) & $75.7(33 \% / \mathrm{m}) \sim 120(42.3 \% / \mathrm{m})$ \\
\hline Ceiling Detector B & $90 \sim 120($ end) & $75.8(33 \% / \mathrm{m}) \sim 120(41.7 \% / \mathrm{m})$ \\
\hline
\end{tabular}

(d) $33 \% / \mathrm{m} \sim 56 \% / \mathrm{m}$ obscuration shall remain.

\begin{tabular}{|l|l|l|}
\hline & UL217 requires(s) & FDS4.0(s) \\
\hline Sidewalls detector A & $80 \sim 120($ end) & $70(33 \% / \mathrm{m}) \sim 120(47.0 \% / \mathrm{m})$ \\
\hline Sidewalls detector B & $80 \sim 120($ end) & $70(33 \% / \mathrm{m}) \sim 120(47.6 \% / \mathrm{m})$ \\
\hline
\end{tabular}

Figure 4a shows the comparison of the obscuration $(\% / \mathrm{m})$ outside the detector and in the sensing chamber at the ceiling detector location D\#1 (see Fig. 3). Using the definition of alarm threshold, the detector activation time was predicted to be approximately 65 seconds, at which time the obscuration outside detector was $20 \% / \mathrm{m}$. Figure $4 \mathrm{~b}$ shows the comparison of the obscuration $(\% / \mathrm{m})$ outside the detector and in the sensing chamber for the sidewall detector location D\#1. The detector activation time is 58 seconds and the obscuration $(\% / \mathrm{m})$ just outside the detector was also over $20 \% / \mathrm{m}$ at this time. 

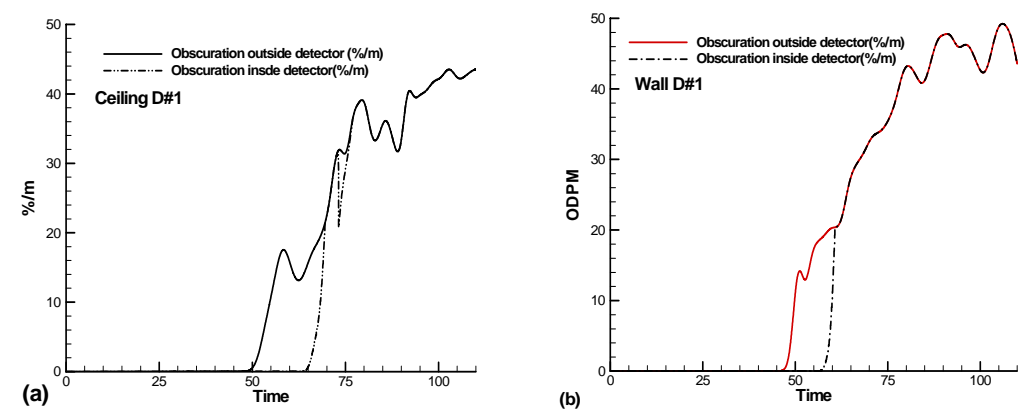

Fig. 4. Comparison of the modeled obscuration outside and inside of the detector for UL217 case D (a. the obscuration at the ceiling location D\#1, b. the obscuration at the sidewall location D\#1).

\section{A Standard Test Fire of the EN54-9/TF4}

A test fire (TF4 in EN54-9 [10]), based on a European standard test for smoke detector activation, was used as the first validation case. TF4 is an open plastic fire, in which a $0.5 \mathrm{~m}$ (length) x $0.5 \mathrm{~m}$ (wide) x $0.25 \mathrm{~m}$ (height) plastic pedestal (Polyurethane Foam Mats) is the fire source. The pedestal is located at the floor center of the enclosure. The dimensions of the enclosure were $9.5 \mathrm{~m}$ (length) x $6.3 \mathrm{~m}$ (wide) x $4.0 \mathrm{~m}$ (height) and all walls, floor and ceiling are considered to be thermally insulated. The smoke detector was located near the ceiling along a $3.0 \mathrm{~m}$ radius from the center point of the ceiling in enclosure $(\mathrm{Z}=3.9 \mathrm{~m}, \mathrm{X}=2.25, \mathrm{Y}=3.15 \mathrm{~m})$. The dimensions of the computational domain are identical to the size of the enclosure and a uniform mesh (80x56x40) was used for simulations. The total run time was 300 seconds. The Heat Release Rate (HRR) was estimated from measurements reported by Ahonen and Sysio [16] and used by Farouk et al. [8]. According to the SFPE handbook [1], the soot yield for the soft polyurethane foam mats can be as large as 0.227 . This value of the soot yield was specified in this study and represents the fraction of soot converted from the fuel. It should be noted that this yield does not account for the processes of soot growth and oxidation, but rather to the net production of the smoke particulate in the fire [17], this value may sensitive to the results.

The smoke particle tracking procedure used in this work requires the initial geometric mean particle diameter (GMD) and the geometric standard deviation (GSD) of the particular fuel. Since the GMD and GSD were not available from experiment [7], these values were obtained from the SFPE handbook [1] and other literature [18,19] as same fuel in this study. The initial GMD was set at $0.34 \mu \mathrm{m}$, after conversion of available particle size data. Since there are no GSD data available for flaming fire of the soft polyurethane foam mats in the SFPE handbook, a GSD of 1.6 was selected from the literature $[18,19]$.

In order to implement the smoke detector activation model, the proportionality constants cited in Eqs. 1and 2 must be known for the smoke detectors. Since the exact proportionality constants for the detectors used in the validation study were not measured, values for unidentified ionization detectors that were measured by Cleary et al.

[4] were used, which are as follows: $\alpha_{1}=2.5, \alpha_{2}=0.76, \beta_{1}=-0.71, \beta_{2}=-0.87$. 


\section{Results and Discussion}

Figure 1a shows the comparison for the EN54-9/TF4 fire of the smoke aerosol particle number concentration at the detector location with experimental data [7]. As can be noted that the prediction agrees with the experimental data reasonably well. The maximum particle number concentration (PNC) of the experimental data is about $1.1 \mathrm{E}+7(\mathrm{~N} / \mathrm{cm} 3)$ at 200 seconds, at which time the prediction of the maximum PNC was close to experimental data. After 200 seconds, the PNC was over predicted. It should be noted that the sensitivity of the results on the initial GMD and GSD needs to be studied.

Figure 1b shows the comparison of the PNC outside of detector and at the sensing chamber. The PNC outside the detector grew quickly when the smoke reached the detector location after 28 seconds. For example, after 30 seconds the PNC was approximately $500000\left(\mathrm{~N} / \mathrm{cm}^{3}\right)$. However, the increase of the PNC in the sensing chamber is delayed, not showing significant changes until 58 seconds. Although, the detector output is a function of the particle number and size distribution, in this study, the smoke detector can be thought to have activated at this time point. Future work will more fully investigate the effect of particle size distribution and number concentration on these predictions. On the other hand, since the PNC is not high enough for significant coagulation before 100 seconds both outside and inside the sensing chamber, this effect is minimal on the detector activation time.
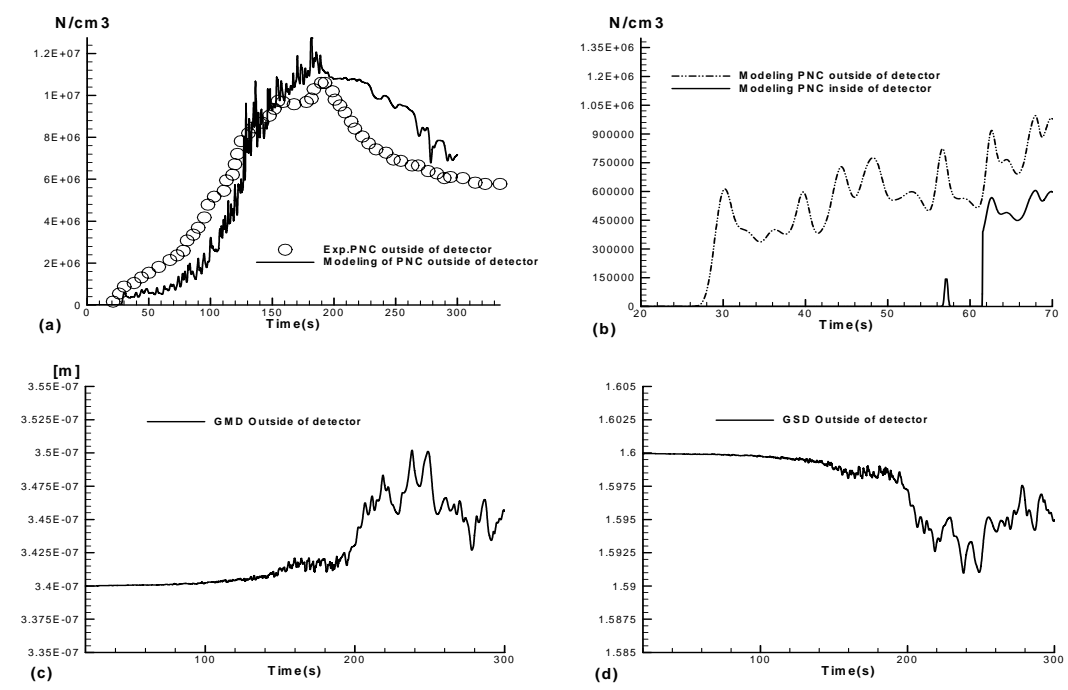

Fig.1. Particle behavior of the EN54-9/TF4 case, (a) comparison of the modeled PNC and experimental data at the detector location, (b) comparison of the modeling PNC inside and outside of the detector within the activation time, (c) modeling of the GMD profile with coagulation effect, (d) modeling of the GSD profile with coagulation effect.

Figure 1c shows the profile of the geometric mean diameter (GMD) with the initial GMD set at $0.34 \mu \mathrm{m}$. The GMD greatly increased after 100 seconds due to the coagulation effect, with the maximum GMD occurring after 200 seconds following the peak PNC. The coagulation process also can be seen in the change in the geometric standard deviation (GSD) as shown in Fig. 1d. The change in GSD occurred after 100 seconds, where the GSD decreased from the initial value. Since this change occurred after detector 
activation was predicted, the change of the GSD was insignificant for predicting detector activation.

\section{CONCLUSIONS}

This study implemented a time lag dynamic response algorithm and a smoke aerosol tracking procedure into a Large Eddy Simulation (LES) fire model. The time lag response and the smoke particle number concentration in and around smoke detector are calculated based on the predicted smoke concentration, the initial particle size and its distribution. Two standard cases were used for verification. For the standard test fire UL217 (D) case, the modeling of the smoke built up was reasonably satisfied by UL217 (D) requirement. With the time lag dynamic response model, the detector activation time could be reasonably estimated. For the standard test fire EN54-9/TF4 case, the predicted particle number concentration (PNC) reasonably agrees with experimental data and the initial geometric mean diameter (GMD) is sensitive for the PNC prediction. The results indicate that before the smoke coagulates process begins at the detector locations, the smoke detector has already activated. Hence, the coagulation process is not significant for modeling smoke detector activation in this case. The smoke detector activation model including time lag dynamic response algorithm and a smoke aerosol tracking procedure can reasonably predict the smoke detector activation time, the particle number and size distribution in the sensing chamber as well as applications of the model for the residential and industrial smoke detector in realistic situations. However, it should be noted that the selected constants (such as soot yield of the fuel; extinction coefficient of the smoke) might sensitive to the results, the sensitivity of these values and the verification of different fire (such as smolder fire) or fuels should be studied in further.

\section{ACKNOWLEDGMENTS}

This work was sponsored by NASA (National Aeronautics and Space Administration) and NIST (National Institute of Standards and Technology). The authors would like to thank Dr. Kevin McGrattan of NIST and Dr. Gary Ruff of NASA for many useful discussion and suggestions.

\section{REFERENCES}

[1] Mulholland, G., "Smoke Production and Properties," The SFPE Handbook of Fire Protection Engineering (2nd ed), P.J. DiNenno (ed.), National Fire Protection Association, Quincy, MA, p. 2/ 221-222, 1995.

[2] Heskestad, G., "Generalized Characteristics of Smoke Entry and Response for Products of Combustion Detectors," Proceeding - $7^{\text {th }}$ International Conference on Problems of Automatic Fire Detection, Hochschule Aachen, March 1975.

[3] Newman, J.S., "Prediction of Fire Detector Response," Fire Safety Journal, Vol: 12, pp. 205-211, 1987.

[4] Cleary, T., Chernovsky, A., Grosshandler, W., and Anderson, M., "Particulate Entry Lags in Spot-type Smoke Detectors," Proceedings of the $6^{\text {th }}$ International Symposium of IAFSS, 1999, pp. 779-790.

[5] Newman, J.S., "Modified Theory for the Characterization of Ionization Smoke Detectors," Proceedings of the Fourth International Symposium of Fire Safety Science, 1989, pp. 785-792. 
[6] Zhang, W., Olenick, S., Roby, R., and Torero, J., "A Smoke Detector Model Algorithm for Large Eddy Simulation Fire Modeling” Submitted to Fire Safety Journal.

[7] Mime, A., Tamm, E., and Sievert, U., "Performance of an Optical and Ionization Smoke Detector Compared to a Wide range Aerosol Spectrometer," Proceedings of the $11^{\text {th }}$ International Conference on Automatic Fire Detection, 1999, Duisburg, Germany, pp. 380-391.

[8] Farouk, B., Mulholland, W., McGrattan, K.B. and Cleary, T.G., "Simulation of Smoke Transport and Coagulation for a Standard Test Fire," Proceedings of the $12^{\text {th }}$ International Conference on Automatic Fire Detection, 2001, NIST, Gaithersburg, MD, USA.

[9] Rexfort, C., "A Contribution to Fire Detection Modeling and Simulation," $P h D$ Dissertation, University of Duisburg-Essen, Germany, March, 2004.

[10] Jackson, M.A. and Robins, I., "Gas Sensing for Fire Detection: Measurements of $\mathrm{CO}, \mathrm{CO}_{2}, \mathrm{H}_{2}, \mathrm{O}_{2}$ and Smoke Density in European Standard Fire Tests,",Fire Safety Journal, 22 (1994), pp. 181-205.

[11] UL217, "Standard for Single and Multiple Station Smoke Detectors," Third Edition, 1985 with updates though February 27, 1989, Underwriters Laboratories, Inc., Northbrook, IL.

[12] Mulholland, G.W. and Croarkin, C., "Specific extinction coefficient of flame generated smoke," Fire and Materials, 24 (5), Sep/Oct 2000, pp. 227-230.

[13] Lee, K. W., Lee, Y. J., and Han, D. S., "The Log-Normal Size Distribution Theory for Brownian Coagulation in the Low Knudsen Number Regime", Journal of Colloid and Interface Science, 188, pp. 486-492, 1997.

[14] Friedlander, S. K., Smoke, Dust and Haze: Fundamentals of Aerosol Dynamics, Oxford University Press, New York, 2000.

[15] Williums, M.M.R., Layolka, S.K., Aerosol Science - Theory and Particle, Pergamon, Press, 1991.

[16] Ahonen, A., and Sysio, P.A., "A Run-in Test Series of a Smoke Test Room-tests according to the Proposal prEN54-9," Research Report 139, Technical research center of Finland, 1983.

[17] “Fire Dynamics Simulator (Version 4.0) - User’s Guide," National Institute of Standards and Technology (NIST), NISTIR 6784, 2003 Ed.

[18] Donnelly, M.K. and Mulholland, G.W., "Particle Size Measurements for Spheres with Diameter of $50 \mathrm{~nm}$ to 400nm,” NISTIR 6935, 2003.

[19] Loepfe, M., Ryser, P., Tompkin, C. and Wieser, "Optical Properties of Fire and Non-fire Aerosols,” Fire safely Journal, 29:185-194 (1997).

[20] D’souza, V.T., Sutula, J.A., Olenick, S.M., Zhang, W. and Roby, R.J., "Predicting Smoke Detector Activation using the Fire Dynamics Simulator," Proceedings of $7^{\text {th }}$ International Symposium of Fire Safety Science, 2002.

[21] Zhang, W., Hamer, A., Klassen, M., Carpenter, D., and Roby, R., “Turbulence Statistics in a Fire Room Modeled by Large Eddy Simulation," Fire Safety Journal, 37 (8):721-752 (2002).

[22] Zhang, W. and Roby, R., "Large Eddy Simulation of Combustion in Compartment Fires,” ASHRAE Transactions, 109, part 2 (4647), pp. 147-157, 2003. 